\title{
Neo-Adjuvant Chemotherapy in Luminal, Node Positive Breast Cancer: Characteristics, Treatment and Oncological Outcomes: A Single Center's Experience
}

(D) Erika Barbieri ${ }^{1}$, (1) Damiano Gentile ${ }^{1,2}$, (1) Alberto Bottini ${ }^{1}$, (1) Andrea Sagona ${ }^{1}$, (D) Wolfgang Gatzemeier ${ }^{1}$, (D) Agnese Losurdo ${ }^{3}$, (D) Bethania Fernandes ${ }^{4}$, (1) Corrado Tinterri ${ }^{1}$

${ }^{1}$ Breast Unit, Institute for Research, Hospitalization and Healthcare (IRCCS) Humanitas Research Hospital, Milan, Italy

${ }^{2}$ Department of Biomedical Sciences, Humanitas University, Milan, Italy

${ }^{3}$ Medical Oncology and Hematology Unit, Institute for Research, Hospitalization and Healthcare (IRCCS) Humanitas Research Hospital, Milan, Italy

${ }^{4}$ Department of Pathology, Institute for Research, Hospitalization and Healthcare (IRCCS) Humanitas Research Hospital, Milan, Italy

\section{ABSTRACT}

Objective: Neo-adjuvant chemotherapy (NAC) is the treatment of choice for patients with locally advanced breast cancer (BC). In luminal-like BC, the decision to administer NAC remains controversial. The purpose of this study was to describe the clinical characteristics, treatment, and oncological outcomes of luminal-like, node positive, BC patients treated with NAC, and to identify independent predictive factors for treatment.

Materials and Methods: All consecutive patients with luminal-like, node positive BC who underwent NAC were retrospectively reviewed. Pathologic complete response (pCR) was defined as no invasive or in situ residual tumor in both breast and axillary nodes (ypT0N0).

Results: A total of 205 luminal-like, node positive BC patients underwent NAC. Overall, 34 (16.6\%) patients showed pCR, 86 (42.0\%) patients underwent breast-conserving surgery (BCS), 119 (58.0\%) patients underwent mastectomy, 130 (63.4\%) patients underwent axillary lymph node dissection (ALND) without prior sentinel lymph node biopsy (SLNB), and 75 (36.6\%) patients underwent breast surgery plus SLNB. Pathologic CR to NAC (29.1\% vs $7.6 \%$ if no $\mathrm{pCR}$, odds ratio $=2.866,95 \%$ confidence interval $=1.296-6.341, \mathrm{p}=0.009$ ) was found to significantly increase the probability to receive $\mathrm{BCS}$. There was no significant difference in terms of disease-free and overall survival between patients with luminal-like, node positive BC receiving BCS or mastectomy ( $\mathrm{p}=0.596, \mathrm{p}=0.134$, respectively), and ALND or SLNB only $(\mathrm{p}=0.661, \mathrm{p}=0.856$, respectively).

Conclusion: Luminal-like, node positive BC presents low pCR rates after NAC. Pre-operative chemotherapy increases the rate of BCS. Pathologic CR has emerged as an independent predictive factor for BCS. In patients with axillary pCR, SLNB is an acceptable procedure not associated with worse oncological outcomes.

Keywords: Chemotherapy, breast cancer, breast-conserving surgery, mastectomy, lymph nodes, dissection

Cite this article as: Barbieri E, Gentile D, Bottini A, Sagona A, Gatzemeier W, Losurdo A, Fernandes B, Tinterri C. Neo-Adjuvant Chemotherapy in Luminal, Node Positive Breast Cancer: Characteristics, Treatment and Oncological Outcomes: A Single Center's Experience. Eur J Breast Health 2021; 17(4): 356-362.

\section{Key Points}

- Despite favorable long-term survival, luminal-like breast cancer is relatively resistant to neo-adjuvant chemotherapy and pathologic complete response is less likely to occur. Therefore, the decision to administer pre-operative chemotherapy remains controversial.

- Although pathologic complete response should not be used as a surrogate endpoint for improved survival, in luminal-like, node positive breast cancer, it has emerged as an independent predictive factor for breast conserving surgery.

- The use of neo-adjuvant chemotherapy in luminal-like, node positive breast cancer allows the performance and increases the rate of breast conserving surgery in patients previously requiring mastectomy.

- In luminal-like, node positive breast cancer patients with axillary pathologic complete response, sentinel lymph node biopsy is a safe and acceptable procedure not associated with worse oncological outcomes. 


\section{Introduction}

The therapeutic algorithm for invasive breast cancer (BC) includes three different treatment modalities: surgery, systemic therapy, and radiation therapy. Traditionally, systemic therapy has been administered to BC patients after surgery, in the adjuvant setting (1). However, the potential role of neo-adjuvant chemotherapy (NAC) - i.e. systemic therapy started before surgery - began to gain importance in breast oncology, and it has been regarded as an equally safe and effective option when compared to adjuvant therapy (2-4). Nowadays, NAC is the treatment of choice for patients with locally advanced $\mathrm{BC}(5,6)$. In this setting, the intent of NAC is to expand surgical options and to improve survival (7). Moreover, indication for NAC has been extended to patients with large, operable BC in order to allow and to increase the rate of breast conserving surgery (BCS), thus avoiding mastectomy $(8,9)$. The next clinical dilemma concerns the optimal management of $\mathrm{BC}$ patients with clinically node positive axilla. Recently, the role of NAC as a means of down-staging the axilla has been investigated, both to avoid the complications of axillary lymph node dissection (ALND) and as a source of prognostic information. Axillary pathologic complete response ( $\mathrm{pCR}$ ) is observed in $20 \%-42 \%$ of node positive patients $(10-12)$ and is associated with a more favourable survival $(12,13)$. It appears rational to conclude that aggressive surgical treatment with ALND might be omitted in patients with axillary pCR $(14,15)$. However, the increasing use of NAC has raised doubts about the optimal approach to the axilla, including accuracy and timing of sentinel lymph node biopsy (SLNB). Furthermore, NAC has been considered a standard therapy only in triple negative (TN) and human epidermal growth factor receptor 2 (HER2) enriched disease, with pCR being more pronounced in these biological sub-types (16). In luminal-like BC, NAC is less effective, achieving lower pCR rates compared to the previously cited more aggressive sub-types, despite high conversion rate from mastectomy to BCS $(17,18)$. Therefore, the decision to administer NAC to luminallike BC patients remains controversial. The purpose of this study was to describe the clinical characteristics, treatment, and oncological outcomes of luminal-like, node positive $\mathrm{BC}$ patients treated with NAC, and to identify independent predictive factors for treatment, resulting in either BCS or mastectomy.

\section{Materials and Methods}

\section{Study design and patient management}

All consecutive patients with luminal-like, node positive BC who underwent NAC at the Breast Unit of Humanitas Clinical and Research Center (Milan, Italy) between January 2008 and December 2019 were retrospectively reviewed. All patients underwent preoperative staging with bilateral breast and axillary ultrasound (US). Pre-operative mammography or magnetic resonance imaging (MRI) of the breast was not mandatory, although, they were performed in the majority of the patients. Diagnosis of invasive $\mathrm{BC}$ with node metastasis was histologically confirmed in all patients by core needle biopsy in both breast and axilla. A multidisciplinary tumor board composed of breast surgeons, oncologists, radiotherapists, radiologists, plastic surgeons, and pathologists discussed the management of every patient. Indication for NAC was: locally advanced, $>2 \mathrm{~cm}$ diameter, luminallike BC with histologically confirmed axillary metastasis. Assessment of response to NAC was performed after each cycle with clinical examination. After three months of NAC, each patient underwent a second bilateral breast and axillary US. All patients underwent postNAC response evaluation by one or a combination of the following radiological examinations: bilateral breast and axillary US, MRI of the breast, or total-body positron emission tomography. All patients underwent either BCS or mastectomy. Indication for conserving surgery or mastectomy was given by the breast surgeon after NAC tumor response evaluation. Conversion rate from mastectomy to BCS was analyzed retrospectively. Regarding the management of the axilla, before 2015, all patients underwent breast surgery plus ALND directly without SLNB. Between 2015 and 2018, all clinically node negative patients underwent breast surgery plus SLNB and in case of sentinel node negativity, ALND was omitted. From December 2018, patients begun to be enrolled in the NEONOD2 prospective clinical trial (19) and in case of micro-metastatic sentinel node, ALND was omitted. The following exclusion criteria were used: patients not treated with NAC; other BC sub-types (TN or HER2 enriched disease); clinical tumor diameter $\leq 2 \mathrm{~cm}$ or not measurable tumor before NAC; patients who did not complete pre-operative staging with both breast and axillary US; patients who did not undergo core needle biopsy of both breast and axilla or post-NAC radiological response evaluation; patients with other prior malignancies; and follow-up $<12$ months. Taxane-based adjuvant chemotherapy was administered to patients who did not achieve pCR and did not complete both anthracycline and taxane-containing regimen before surgery. Adjuvant endocrine treatment was administered to all patients after surgery and adjuvant radiotherapy was recommended for all patients who underwent BCS. Follow-up was performed every six months, including physical examination, routine biochemistry, mammography, and breast and axilla US. Abdominal US and chest X-ray was prescribed annually. Each patient gave informed consent for the operation and for clinical data acquisition.

\section{Definitions}

In 2011, the St. Gallen expert consensus panel adopted a sub-typebased approach for the treatment of early $\mathrm{BC}$ using levels of estrogen receptor (ER), progesterone receptor (PgR), Ki67, and HER2 expression (20). In our study and in line with the 2013 St. Gallen update (21), luminal A-like BC was defined as ER positive, PgR positive (cut-point $\geq 20 \%$ ), HER2 negative, Ki67 'low' $<14 \%$, whereas luminal B-like BC was defined as ER positive, PgR negative or low (cut-point $<20 \%$ ), HER2 negative, Ki67 'high' $\geq 14 \%$. Additionally, luminal B-like BC was defined as ER-positive, highly proliferating disease with high histological tumor grade (G3) $(22,23)$. However, none of these classification systems could produce a strong consensus in sub-dividing luminal-like BC (24). Despite ER being bimodally expressed, thus creating an important cut-off point, proliferationrelated genes are expressed along a unimodal continuum, making it extremely difficult to apply a significant cut-off point (25). HER2 status was assessed by immunohistochemistry and defined as negative if the score was $0 / 1+$, equivocal if the score was $2+$, or positive if the score was 3+. Equivocal cases were further assessed by fluorescent in situ hybridization, according to the recommendations of the American Society of Clinical Oncology/College of American Pathologists (ASCO/CAP) (26). Tumor staging was defined according to the American Joint Committee on Cancer (AJCC) tumor-node-metastasis (TNM) cancer staging system (AJCC Cancer Staging Manual, $8 \mathrm{t}^{\mathrm{h}}$ edition) (27). Tumor response rate to NAC was defined as the calculated percent rate of breast tumor and axillary node size reduction between baseline and after systemic therapy. The tumor response rate was calculated according to the Response Evaluation Criteria in Solid Tumors (RECIST) 1.1 criteria (28). Pathologic CR was defined as no invasive or non-invasive residual tumor in both breast and axillary 
nodes (ypT0 N0), excluding patients with pathological stage ypTis. Disease-free survival (DFS) was defined as the period from the date of surgery to the date of any tumor progression, including loco-regional recurrence or distant metastasis. Overall survival (OS) was defined as the time interval from surgery to death from any cause or last followup.

\section{Statistical analysis}

Patients were selected from the institutional prospectively maintained database and retrospectively analyzed. Categorical variables were compared using the chi-square test or Fisher's exact test, as appropriate. The Kaplan-Meier method was used to estimate the recurrence and survival probabilities and the log-rank test was used to compare two different groups of axillary treatment (SLNB-only vs ALND). Last follow-up was updated up to December $1^{\text {st }}, 2020$. Follow-up was $\geq 12$ months in all luminal-like, node positive BC patients and no patient was lost to follow-up. The multivariate analysis was performed using a logistic regression model to identify independent predictors of treatment for luminal-like, node positive BC. The multivariate analysis included any variable associated with the result at the univariate analysis (inclusion cut-off value $\mathrm{p}<0.10$ ). Statistical significance was set at $\mathrm{p}<0.050$. Data analyses and figures were performed with IBM SPSS, version 25.0 (IBM Inc., Armonk, NY, USA).

\section{Results}

\section{Patients' characteristics}

Between January 2008 and December 2019, 5,739 patients with luminal-like BC underwent surgical treatment at the Breast Unit of Humanitas Clinical and Research Center (Milan, Italy). Of these patients, 205 luminal-like, node positive patients underwent NAC before surgery, of whom $124(2.2 \%)$ and 81 (1.4\%) were luminal A-like and luminal B-like, respectively. The mean age of the patients was 54.8 years (range, 30-77), and $136(66.3 \%)$ patients were postmenopausal. Bilateral mammography and MRI of the breast were performed in 191 (93.2\%) and 89 (43.4\%) patients, respectively. Before NAC, the mean diameter of breast tumor was $32 \mathrm{~mm}$ (range, 21-80), $148(72.2 \%)$ patients were affected by cT2 BC, and $47(22.9 \%)$ and $20(9.8 \%)$ patients were affected by multifocal and multicentric tumors, respectively. Regarding NAC treatment protocol, 69 (33.7\%) patients received only anthracycline for four cycles, while $136(66.3 \%)$ patients received both anthracycline and taxanes. After NAC, the mean diameter of the breast tumor was $22 \mathrm{~mm}$ (range, 0-75). Out of these 205 patients, 34 (16.6\%) showed pCR. Characteristics of these patients are summarized in Table 1.

\section{Treatment of the breast and axilla}

Overall, 86 (42.0\%) patients underwent BCS, while 119 (58.0\%) patients underwent mastectomy. Thirty-three (16.1\%) patients who were initially candidates for mastectomy, were subsequently treated with BCS. Regarding the management of the axilla, 130 (63.4\%) patients underwent breast surgery plus ALND without SLNB, and 75 (36.6\%) underwent breast surgery plus SLNB. Of the latter group, 41 (54.7\%) had positive sentinel node and underwent subsequent ALND, while eight (10.7\%) patients and $26(34.6 \%)$ patients had micrometastatic and negative sentinel node, respectively and thus ALND was omitted. Regarding adjuvant treatment, 16 (7.8\%) patients and $182(88.8 \%)$ patients underwent post-operative systemic therapy and radiotherapy, respectively. On multivariate analysis, one independent
Table 1. Characteristics of 205 patients with luminal, node positive breast cancer treated with neo-adjuvant chemotherapy

Characteristics

Number (\%)/mean (SD)

\section{Patients}

Age (years)

Post-menopausal

$54.8(11.9)$

Pre-operative staging

$136(66.3 \%)$

Mammography

$191(93.2 \%)$

US*

$205(100 \%)$

Biopsy $^{\circ}$

$205(100 \%)$

MRI

89 (43.4\%)

Dimension pre-NAC (mm)

$32(17.1)$

Stage pre-NAC

- $\mathrm{cT} 2$

$148(72.2 \%)$

- $\mathrm{CT} 3$

$35(17.1 \%)$

- CT4

$22(10.7 \%)$

$-\mathrm{cN} 1$

$205(100 \%)$

NAC with anthracycline only

$69(33.7 \%)$

NAC with anthracycline and taxanes

$136(66.3 \%)$

Tumor

Multifocal

47 (22.9\%)

Multicentric

20 (9.8\%)

\section{Sub-type}

- Luminal A-like

124 (60.5\%)

- Luminal B-like

81 (39.5\%)

\section{Histotype}

- Ductal

188 (91.7\%)

- Lobular

$11(5.4 \%)$

- Mucinous

$6(2.9 \%)$

Grade G3

56 (27.3\%)

Vascular invasion

74 (36.1\%)

Single nodule

151 (73.7\%)

PCR to NAC

$34(16.6 \%)$

Dimension post-NAC (mm)

22 (18.9)

\section{Stage post-NAC}

- урт0

34 (16.6\%)

- урT1а

14 (6.8\%)

- урT1b

$23(11.2 \%)$

- урT1с

$42(20.5 \%)$

- урт2

$78(38.1 \%)$

- урТ3

$14(6.8 \%)$

- ypNO

$44(21.5 \%)$

ypNmi

8 (3.9\%)

-ypN1

47 (22.9\%)

- ypN2

77 (37.6\%)

- ypN3 
Table 1. Continued

\section{Characteristics}

\section{Surgical treatment}

- BCS

- Mastectomy

- SLNB not followed by ALND

- SLNB followed by ALND

Post-operative treatment

- $\mathrm{CHT}$

- Radiotherapy

- Endocrine

SD: Standard deviation, US*: Breast and axillary ultrasound, Biopsy: Breast and axillary ultrasound-guided biopsy, MRI: Magnetic resonance imaging, NAC: Neo-adjuvant chemotherapy, CHT: Chemotherapy, pCR: Pathological in-breast and axillary response to neo-adjuvant chemotherapy, BCS: Breast conserving surgery, SLNB: Sentinel lymph node biopsy, ALND: Axillary lymph node dissection

Pathologic CR to NAC $(29.1 \%$ vs $7.6 \%$ if pCR to NAC, odds ratio $(\mathrm{OR})=2.866,95 \%$ confidence interval $(95 \% \mathrm{CI})=1.296-6.341, \mathrm{p}$ $=0.009)$ was found to significantly increase the probability to receive BCS for luminal-like, node positive BC. Treatment details, univariate and multivariate analyses are summarized in Tables 1 and 2 .

\section{Oncological outcomes}

After a mean follow-up of 53 months, 18/205 (8.8\%) patients developed recurrence. In the BCS group, $4 / 86$ (4.7\%) and 6/86 (7.0\%) had loco-regional recurrence and distant metastases, respectively. In the mastectomy group, $2 / 119(1.7 \%)$ and $6 / 119$ (5.0\%) had locoregional recurrence and distant metastases, respectively. All the patients in the BCS group who had loco-regional recurrence underwent salvage mastectomy. Patients with distant metastases were treated with systemic therapy. Overall, 15/205 (7.3\%) patients died; 12 patients suffered a BC related death, while three patients died for other reasons. Moreover, the oncological outcomes of conservative and radical breast and axillary treatment were analyzed and compared. There was no significant difference in terms of DFS and OS between patients with luminal-like, node positive $\mathrm{BC}$ receiving $\mathrm{BCS}$ or mastectomy ( $\mathrm{p}=0.596, \mathrm{p}=0.134$, respectively), and ALND or SLNB only ( $\mathrm{p}=$ $0.661, p=0.856$, respectively) after NAC. Oncological outcomes are summarized in Table 3 and Figures 1 and 2.

\section{Discussion and Conclusion}

The ability of NAC to evaluate the in vivo chemo-sensitivity of primary $\mathrm{BC}$ has emerged as a major prognostic tool in understanding individual patient outcome. Pathologic CR has been associated with improved long-term oncological outcomes in virtually every study and has emerged as a surrogate endpoint for survival in several NAC trials (29). However, the prognosis and responses to NAC differ according to the biological sub-type of BC. Generally, patients with luminal-like $\mathrm{BC}$ show good long-term oncological results, whereas those with TN and HER2-enriched disease have poor outcomes (16). Interestingly, it appears that the most aggressive biologic and tumor-related factors (high grade, high Ki-67, ER negativity) are more closely associated with achieving $\mathrm{pCR}$. On the other hand, luminal-like $\mathrm{BC}$ is relatively resistant to NAC and $\mathrm{pCR}$ is less likely to occur, despite favorable

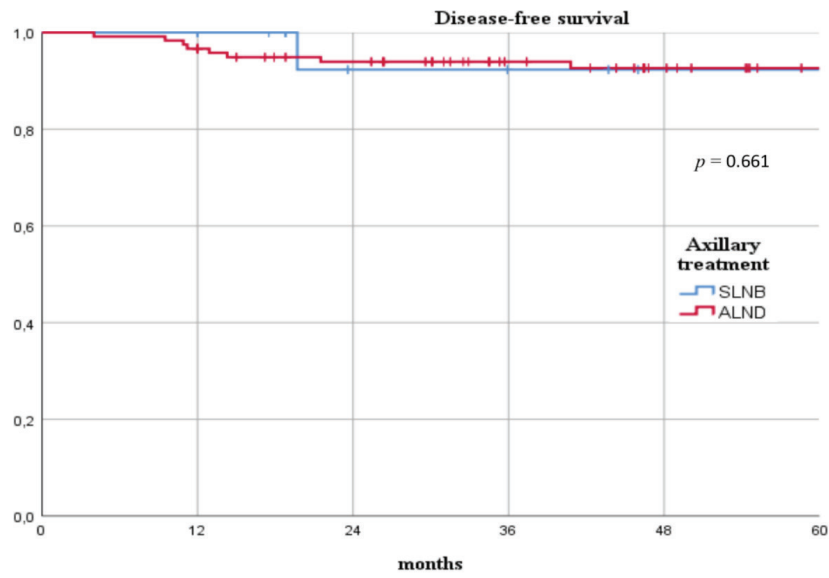

Figure 1. Disease-free survival of luminal, node positive breast cancer patients treated with neo-adjuvant chemotherapy according to axillary treatment

This figure depicts the recurrence curves of luminal, node positive breast cancer patients according to axillary treatment after neoadjuvant chemotherapy.

ALND: Axillary lymph node dissection, SLNB: Sentinel lymph node biopsy only

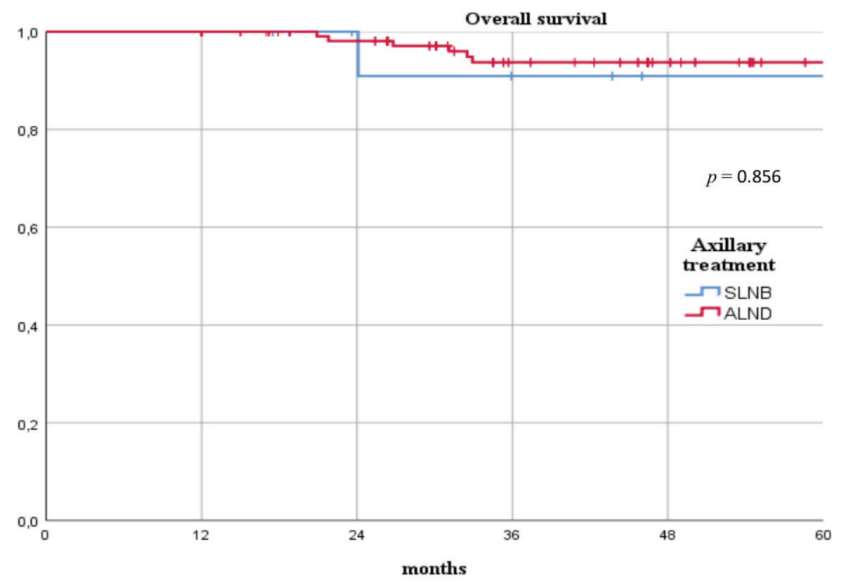

Figure 2. Overall survival of luminal, node positive breast cancer patients treated with neo-adjuvant chemotherapy according to axillary treatment

This figure depicts the survival curves of luminal, node positive breast cancer patients according to axillary treatment after neo-adjuvant chemotherapy.

ALND: Axillary lymph node dissection, SLNB: Sentinel lymph node biopsy only

long-term survival. A meta-analysis of 30 studies including 11,695 patients evaluating pCR after NAC found an overall pooled estimate of pCR of $19.2 \%$ (30). The probability of achieving pCR was seven times higher for HER2-enriched disease and five times higher for $\mathrm{TN}$ disease when compared to luminal-like BC. While pCR rates may be low in luminal-like sub-type, survival outcomes remain good, mainly because of favorable biological characteristics and benefits from adjuvant endocrine treatment. In 2012, a pooled analysis of 6,377 patients treated with anthracycline and taxane-based NAC showed that, in luminal-like BC, pCR did not have predictive power in terms of DFS and OS (18). Our study confirms the low pCR rate (16.6\%) after NAC in luminal-like sub-type, and even though pCR 
Table 2. Predictors of treatment (breast conserving surgery versus mastectomy) in luminal, node positive breast cancer patients treated with neo-adjuvant chemotherapy

\begin{tabular}{|c|c|c|c|c|}
\hline \multirow{2}{*}{ Characteristics } & \multirow{2}{*}{$\begin{array}{c}\text { BCS } \\
(\mathrm{n}=86) \\
\text { Tot. }(\%)\end{array}$} & \multirow{2}{*}{$\begin{array}{l}\text { Mastectomy } \\
(\mathrm{n}=119) \\
\text { Tot. }(\%)\end{array}$} & \multirow{2}{*}{$\begin{array}{l}\text { Univariate } \\
\text { analysis } \\
\text { p-value }\end{array}$} & \multirow{2}{*}{$\begin{array}{l}\text { Multivariable analysis } \\
\text { p-value OR ( } 95 \% \mathrm{CI})\end{array}$} \\
\hline & & & & \\
\hline \multicolumn{5}{|l|}{ Demographic } \\
\hline $\begin{array}{l}\text { Age (years) } \\
-<55 \\
-\geq 55\end{array}$ & $\begin{array}{l}47(54.7 \%) \\
39(45.3 \%)\end{array}$ & $\begin{array}{l}78(65.6 \%) \\
41(34.4 \%)\end{array}$ & $\begin{array}{c}0.165 \\
-\end{array}$ & - \\
\hline $\begin{array}{l}\text { Post-menopausal } \\
\text { - Yes } \\
\text { - No }\end{array}$ & $\begin{array}{l}60(69.8 \%) \\
26(30.2 \%)\end{array}$ & $\begin{array}{l}76(63.9 \%) \\
43(36.1 \%)\end{array}$ & $\begin{array}{c}0.736 \\
-\end{array}$ & - \\
\hline \multicolumn{5}{|l|}{ Pre-operative staging } \\
\hline $\begin{array}{l}\text { Stage pre-NAC } \\
\text { - cT2 } \\
\text { - cT3-4 }\end{array}$ & $\begin{array}{l}64(74.4 \%) \\
22(25.6 \%)\end{array}$ & $\begin{array}{l}84(70.6 \%) \\
35(29.4 \%)\end{array}$ & $\begin{array}{c}0.752 \\
-\end{array}$ & - \\
\hline $\begin{array}{l}\text { NAC } \\
\text { - Anthracycline and taxanes } \\
\text { - Anthracycline only }\end{array}$ & $\begin{array}{l}61(70.9 \%) \\
25(29.1 \%)\end{array}$ & $\begin{array}{l}75(63.0 \%) \\
44(37.0 \%)\end{array}$ & $\begin{array}{c}0.248 \\
-\end{array}$ & - \\
\hline \multicolumn{5}{|l|}{ Tumor histotype } \\
\hline $\begin{array}{l}\text { - Ductal } \\
\text { - Lobular } \\
\text { - Mucinous } \\
\text { Vascular invasion }\end{array}$ & $\begin{array}{c}79 \text { (91.9\%) } \\
5(5.8 \%) \\
2(2.3 \%)\end{array}$ & $\begin{array}{c}109(91.6 \%) \\
6(5.0 \%) \\
4(3.4 \%)\end{array}$ & $\begin{array}{c}0.872 \\
- \\
-\end{array}$ & - \\
\hline $\begin{array}{l}\text { - Yes } \\
\text { - No } \\
\text { Sinale nodule }\end{array}$ & $\begin{array}{l}36(41.9 \%) \\
50(58.1 \%)\end{array}$ & $\begin{array}{l}38(31.9 \%) \\
81(68.1 \%)\end{array}$ & $\begin{array}{c}0.193 \\
-\end{array}$ & - \\
\hline $\begin{array}{l}\text { Single nodule } \\
\text { - Yes }\end{array}$ & & & & \\
\hline - No & $\begin{array}{l}71(82.6 \%) \\
15(17.4 \%)\end{array}$ & $\begin{array}{l}80(67.2 \%) \\
39(32.8 \%)\end{array}$ & $\begin{array}{c}0.002^{\mathrm{a}} \\
-\end{array}$ & $\begin{array}{c}0.2210 .628(0.298-1.324) \\
-\end{array}$ \\
\hline $\begin{array}{l}\text { - Yes } \\
\text { - No }\end{array}$ & $\begin{array}{l}25(29.1 \%) \\
61(70.9 \%)\end{array}$ & $\begin{array}{c}9(7.6 \%) \\
110(92.4 \%)\end{array}$ & $\begin{array}{c}0.001^{\mathrm{a}} \\
-\end{array}$ & $\begin{array}{c}0.009^{a} 2.866(1.296-6.341) \\
-\end{array}$ \\
\hline
\end{tabular}

BCS: Breast conserving surgery, n: Number, Tot.: Total, OR: Odds ratio, Cl: Confidence interval, NAC: Neo-adjuvant chemotherapy, pCR: Pathological inbreast and axillary response to neo-adjuvant chemotherapy, a: Statistically significant

should not be used as a surrogate endpoint for improved survival in this setting, it has emerged as an independent predictive factor for breast conservation.

Neo-adjuvant chemotherapy is the treatment of choice for patients with large, operable BC. Its main effects include: decreasing the size and cellularity of the tumor; reducing the surgical resection range; and increasing the rate of BCS conversion, thus improving the cosmetic outcomes and quality of life. The National Surgical Adjuvant Breast and Bowel Project (NSABP) B-18 trial evaluated the facilitation of breast conservation in the NAC setting, and found increased rates of breast conservation in the NAC arm $(67.8 \%$ vs $59.8 \%$ ) (4). Additionally, results of the European Organization Research and Treatment of Cancer (EORTC) Trial 10902 showed a significant reduction in clinical tumor diameter to less than 2 $\mathrm{cm}$ from $14 \%$ at primary diagnosis to $47 \%$ after NAC, with a BCS conversion rate of $23 \%$ (31). In our study we retrospectively multifocality, and multicentricity and their surgical indication before NAC. The use of pre-operative systemic treatment allowed the performance of conserving surgery in 33 additional patients previously requiring mastectomy, increasing the rate of BCS by $16.1 \%$.

Traditionally, all patients who presented with clinically node positive BC were recommended complete ALND, in order to eradicate the nodal basin of any lymph node disease, reducing the risk of loco-regional recurrence, and providing full pathologic nodal staging. Complete ALND remains an acceptable option for some patients with axillary metastases identified at the time of diagnosis. However, for patients receiving NAC, there is an increasing body of evidence supporting a more conservative axillary approach in patients with good clinical response. The American College of Surgeon Oncology Group (ACOSOG) Z1071 trial reported that approximately $65 \%$ of patients with HER2-enriched disease, $50 \%$ of patients with TN disease, and $21 \%$ of patients with luminal- 
Table 3. Comparison of disease-free and overall survival of luminal, node positive breast cancer patients undergoing conservative or radical breast and axillary treatment after neo-adjuvant chemotherapy

\begin{tabular}{|c|c|c|c|c|c|c|}
\hline \multirow{2}{*}{ Outcomes } & $\begin{array}{c}\text { BCS } \\
(n=86)\end{array}$ & $\begin{array}{c}\text { Mastectomy } \\
(n=119)\end{array}$ & \multirow{2}{*}{ p-value } & $\begin{array}{c}\text { ALND } \\
(n=171)\end{array}$ & $\begin{array}{c}\text { SLNB } \\
(n=34)\end{array}$ & \multirow{2}{*}{ p-value } \\
\hline & \multicolumn{2}{|c|}{ Mean (SD) } & & \multicolumn{2}{|c|}{ Mean (SD) } & \\
\hline \multicolumn{7}{|l|}{ DFS rate } \\
\hline - 1-year & $95.1 \%$ & $98.8 \%$ & & $96.7 \%$ & $100 \%$ & \multirow{3}{*}{0.661} \\
\hline - 3-year & $93.2 \%$ & $94.7 \%$ & 0.596 & $94.0 \%$ & $92.3 \%$ & \\
\hline - 5-year & $92.6 \%$ & $92.9 \%$ & & $92.6 \%$ & $92.3 \%$ & \\
\hline \multicolumn{7}{|l|}{ OS rate } \\
\hline - 1-year & $100 \%$ & $100 \%$ & & $100 \%$ & $100 \%$ & \multirow{3}{*}{0.856} \\
\hline - 3-year & $90.7 \%$ & $95.3 \%$ & 0.134 & $93.7 \%$ & $90.9 \%$ & \\
\hline - 5-year & $90.7 \%$ & $95.3 \%$ & & $93.7 \%$ & $90.9 \%$ & \\
\hline
\end{tabular}

BCS: Breast conserving surgery, n: Number, ALND: Axillary lymph node dissection, SLNB: Sentinel lymph node biopsy only, SD: Standard deviation, DFS: Disease-free survival, OS: Overall survival

like BC had pCR in the axilla (32). The benefits to avoiding unnecessary ALND for patients with strong response to NAC are clear, with a substantial reduction of the morbidity associated with the procedure, which may include paresthesias, pain, wound infection, seromas, and lymphedema. In our study, we showed that there was no significant difference in terms of DFS and OS between patients with luminal-like, node positive $\mathrm{BC}$ receiving ALND or SLNB only after NAC.

It is necessary to underline that our study has some limitations. Firstly, this is a single center study, subject to limitations due to its retrospective design, using observational data collected at a specific moment. Secondly, the evaluation of conversion from mastectomy to BCS was performed retrospectively. Despite these limitations, this study also presents several strong points. Definitions were clearly stated and strict inclusion criteria were used for the selection of a homogeneous group of luminal-like, node positive BC. All patients underwent pre-operative radiological staging, core needle biopsy in both breast and axilla, and post-NAC radiological response evaluation. Moreover, no patient was lost to follow-up.

In conclusion, our study confirms the low pCR rate after NAC in luminal-like, node positive BC. Pre-operative systemic treatment increases the rate of BCS. Although pCR should not be used as a surrogate endpoint for improved survival in luminal-like BC, it has emerged as an independent predictive factor for BCS. Additionally, in patients with axillary pCR, SLNB is a safe and acceptable procedure not associated with worse oncological outcomes.

Ethics Committee Approval: The present study complied with the guidelines for human studies. The research was conducted ethically in accordance with the World Medical Association Declaration of Helsinki. The Institutional Review Board of Humanitas University Research Ethics Committee approved this retrospective study (no: H20-12-NAC, date: 12.12.2020).

Informed Consent: Each patient provided informed consent for operation and clinical data acquisition.

Peer-review: Externally peer-reviewed.

\section{Authorship Contributions}

Surgical and Medical Practices: E.B., D.G., A.B., A.S., W.G., A.L., B.F., C.T., Concept: E.B., D.G., A.S., A.L., C.T., Design: E.B., D.G., A.S., A.L., C.T., Data Collection and/or Processing: E.B., D.G., A.B., A.S., W.G., A.L., B.F., C.T., Literature Search: E.B., D.G., A.B., A.S., W.G., B.F., Writing: E.B., D.G., A.B., A.S., W.G., B.F.

Conflict of Interest: No conflict of interest was declared by the authors.

Financial Disclosure: This research did not receive any specific grant from funding agencies in the public, commercial, or not-for profit sectors.

\section{References}

1. DeVita VT, Chu E. A history of cancer chemotherapy. Cancer Res 2008; 68: 8643-8653. (PMID: 18974103) [Crossref]

2. Van Nes JGH, Putter H, Julien JP, et al. Preoperative chemotherapy is safe in early breast cancer, even after 10 years of follow-up; Clinical and translational results from the EORTC trial 10902. Breast Cancer Res Treat 2009; 115: 101-113. (PMID: 18484198) [Crossref]

3. Mauri D, Pavlidis N, Ioannidis JPA. Neoadjuvant versus adjuvant systemic treatment in breast cancer: A meta-analysis. J Natl Cancer Inst 2005; 97: 188-194. (PMID: 15687361) [Crossref]

4. Rastogi P, Anderson SJ, Bear HD, et al. Preoperative chemotherapy: updates of national surgical adjuvant breast and bowel project protocols B-18 and B-27. J Clin Oncol 2008; 26: 778-785. (PMID: 18258986) [Crossref]

5. Choudhary P, Gogia A, Deo S, Mathur S, Sharma D. Neoadjuvant chemotherapy in locally advanced breast cancer: Clinicopathological characteristics and correlation with pathological complete response. J Clin Oncol 2020; 38: 12658-12658.

6. Kaufmann M, Von Minckwitz G, Mamounas EP, Cameron D, Carey LA, Cristofanilli M, et al. Recommendations from an international consensus conference on the current status and future of neoadjuvant systemic therapy in primary breast cancer. Ann Surg Oncol 2012; 19: 1508-1516. (PMID: 22193884) [Crossref]

7. Specht J, Gralow JR. Neoadjuvant chemotherapy for locally advanced breast cancer. Semin Radiat Oncol 2009; 19: 222-228. (PMID: 19732686) [Crossref]

8. Vugts G, Maaskant-Braat AJG, de Roos WK, Voogd AC, Nieuwenhuijzen GAP. Management of the axilla after neoadjuvant chemotherapy for 
clinically node positive breast cancer: a nationwide survey study in The Netherlands. Eur J Surg Oncol 2016; 42: 956-964. (PMID: 27107791) [Crossref]

9. Golshan M, Cirrincione CT, Sikov WM, Berry DA, Jasinski S, Weisberg TF, et al; Alliance for Clinical Trials in Oncology. Impact of neoadjuvant chemotherapy in stage II-III triple negative breast cancer on eligibility for breast-conserving surgery and breast conservation rates: surgical results from CALGB 40603 (Alliance). Ann Surg. 2015; 262: 434-439; discussion 438-439. (PMID: 26222764) [Crossref]

10. Alvarado R, Yi M, Le-Petross H, Gilcrease M, Mittendorf EA, Bedrosian I, et al. The role for sentinel lymph node dissection after neoadjuvant chemotherapy in patients who present with node-positive breast cancer. Ann Surg Oncol 2012; 19: 3177-3184. (PMID: 22772869) [Crossref]

11. Koolen BB, Valdés Olmos RA, Wesseling J, Vogel WV, Vincent AD, Gilhuijs KG, et al. Early assessment of axillary response with 18F-FDG PET/CT during neoadjuvant chemotherapy in stage II-III breast cancer: Implications for surgical management of the axilla. Ann Surg Oncol 2013; 20: 2227-2235. (PMID: 23456316) [Crossref]

12. Straver ME, Rutgers EJT, Russell NS, Oldenburg HS, Rodenhuis $\mathrm{S}$, Wesseling J, et al. Towards rational axillary treatment in relation to neoadjuvant therapy in breast cancer. Eur J Cancer 2009; 45: 2284-2292. (PMID: 19464164) [Crossref]

13. Hennessy BT, Hortobagyi GN, Rouzier R, Kuerer H, Sneige N, Buzdar $\mathrm{AU}$, et al. Outcome after pathologic complete eradication of cytologically proven breast cancer axillary node metastases following primary chemotherapy. J Clin Oncol 2005; 23: 9304-9311. (PMID: 16361629) [Crossref]

14. Mamtani A, Barrio AV, King TA, Van Zee KJ, Plitas G, Pilewskie M, et al. How often does neoadjuvant chemotherapy avoid axillary dissection in patients with histologically confirmed nodal metastases? results of a prospective study. Ann Surg Oncol 2016; 23: 3467-3474. (PMID: 27160528) [Crossref]

15. Kahler-Ribeiro-Fontana S, Pagan E, Magnoni F, Vicini E, Morigi C, Corso G, et al. Long-term standard sentinel node biopsy after neoadjuvant treatment in breast cancer: a single institution ten-year follow-up. Eur J Surg Oncol 2020; 47: 804-812. (PMID: 33092968) [Crossref]

16. Harbeck N, Gluz O. Neoadjuvant therapy for triple negative and HER2positive early breast cancer. Breast. 2017; 34(Suppl 1): S99-S103. (PMID: 28666920) [Crossref]

17. Rouzier R, Perou CM, Symmans WF, Ibrahim N, Cristofanilli M, Anderson K, et al. Breast cancer molecular subtypes respond differently to preoperative chemotherapy. Clin Cancer Res 2005; 11: 5678-5685. (PMID: 16115903) [Crossref]

18. Von Minckwitz G, Untch M, Blohmer JU, Costa SD, Eidtmann H, Fasching PA, et al. Definition and impact of pathologic complete response on prognosis after neoadjuvant chemotherapy in various intrinsic breast cancer subtypes. J Clin Oncol 2012; 30: 1796-1804. (PMID: 22508812) [Crossref]

19. Tinterri C, Canavese G, Bruzzi P, Dozin B. NEONOD2: Rationale and design of a multicenter non-inferiority trial to assess the effect of axillary surgery omission on the outcome of breast cancer patients presenting only micrometastasis in the sentinel lymph node after neoadjuvant chemotherapy. Contemp Clin Trials Commun 2020; 17: 100496. (PMID: 31872159) [Crossref]
20. Goldhirsch A, Wood WC, Coates AS, Gelber RD, Thürlimann B, Senn HJ. Strategies for subtypes-dealing with the diversity of breast cancer: Highlights of the St Gallen international expert consensus on the primary therapy of early breast cancer 2011. Ann Oncol 2011; 22: 1736-1747. (PMID: 21709140) [Crossref]

21. Goldhirsch A, Winer EP, Coates AS, Gelber RD, Piccart-Gebhart M, Thürlimann B, et al; Panel members. Personalizing the treatment of women with early breast cancer: Highlights of the St Gallen international expert consensus on the primary therapy of early breast cancer 2013. Ann Oncol 2013; 24: 2206-2223. (PMID: 23917950) [Crossref]

22. Wirapati P, Sotiriou C, Kunkel S, Farmer P, Pradervand S, Haibe-Kains $\mathrm{B}$, et al. Meta-analysis of gene expression profiles in breast cancer: toward a unified understanding of breast cancer subtyping and prognosis signatures. Breast Cancer Res 2008; 10: R65. [Crossref]

23. Sotiriou C, Pusztai L. Gene-expression signatures in breast cancer. N Engl J Med 2009; 360: 790-800. (PMID: 19228622) [Crossref]

24. MacKay A, Weigelt B, Grigoriadis A, Kreike B, Natrajan R, A'Hern R, et al. Microarray-based class discovery for molecular classification of breast cancer: Analysis of interobserver agreement. J Natl Cancer Inst 2011; 103: 662-673. (PMID: 21421860) [Crossref]

25. Collins LC, Botero ML, Schnitt SJ. Bimodal frequency distribution of estrogen receptor immunohistochemical staining results in breast cancer: an analysis of 825 cases. Am J Clin Pathol 2005; 123: 16-20. (PMID: 15762275) [Crossref]

26. Wolff AC, McShane LM, Hammond MEH, Harvey BE, Mangu PB, Bartlett JMS, et al. Human epidermal growth factor receptor 2 testing in breast cancer: American Society of Clinical Oncology/College of American Pathologists Clinical Practice Guideline Focused Update. Arch Pathol Lab Med 2018; 142: 1364-1382. (PMID: 29846122) [Crossref]

27. Giuliano AE, Edge SB, Hortobagyi GN. Eighth edition of the AJCC cancer staging manual: breast cancer. Ann Surg Oncol 2018; 25: 17831785. (PMID: 29671136) [Crossref]

28. Schwartz LH, Litière S, De Vries E, Ford R, Gwyther S, Mandrekar S, et al. RECIST 1.1 - update and clarification: from the RECIST committee. Eur J Cancer 2016; 62: 132-137. (PMID: 27189322) [Crossref]

29. Teshome M, Kuerer HM. Breast conserving surgery and locoregional control after neoadjuvant chemotherapy. Eur J Surg Oncol 2017; 43: 865-874. (PMID: 28284519) [Crossref]

30. Houssami N, MacAskill P, Von Minckwitz G, Marinovich ML, Mamounas E. Meta-analysis of the association of breast cancer subtype and pathologic complete response to neoadjuvant chemotherapy. Eur J Cancer 2012; 48: 3342-3354. (PMID: 22766518) [Crossref]

31. Van der Hage JA, Van de Velde CJH, Julien JP, Tubiana-Hulin M, Vandervelden C, Duchateau L. Preoperative chemotherapy in primary operable breast cancer: results from the European Organization for Research and Treatment of Cancer Trial 10902. J Clin Oncol 2001; 19: 4224-4237. (PMID: 11709566) [Crossref]

32. Boughey JC, McCall LM, Ballman KV, Mittendorf EA, Ahrendt GM, Wilke LG, et al. Tumor biology correlates with rates of breastconserving surgery and pathologic complete response after neoadjuvant chemotherapy for breast cancer findings from the ACOSOG Z1071 (alliance) prospective multicenter clinical trial. Ann Surg 2014; 260: 608616. (PMID: 25203877) [Crossref] 